\title{
Student Ideas around Vector Decomposition in the Upper Division
}

\author{
Anna Turnbull, ${ }^{1}$ Leanne Doughty, ${ }^{2}$ Vashti Sawtelle, ${ }^{1,2}$ and Marcos D. Caballero ${ }^{2,3}$ \\ ${ }^{1}$ Lyman Briggs College, Michigan State University, East Lansing, MI 48824 \\ ${ }^{2}$ Department of Physics and Astronomy, Michigan State University, East Lansing, MI 48824 \\ ${ }^{3}$ CREATE for STEM Institute, Michigan State University, East Lansing, MI 48824
}

(Dated: September 25, 2015)

\begin{abstract}
Vector decomposition is a skill required across physics contexts and levels. In this paper, we present our analysis of 463 student responses to an item on the Colorado Classical Mechanics/Math Methods Instrument that assesses student proficiency with vector decomposition in multiple coordinate systems. We offer separate categorizations for students' final expressions and their approaches to determining those expressions. The intersection between approaches and final expressions suggests that certain approaches are more productive. We discuss these intersections and the differences between students' responses in Cartesian and polar coordinate systems.
\end{abstract}

PACS numbers: 01.40.Fk

\section{INTRODUCTION}

In recent years, there has been an emphasis on understanding and improving student learning in upper-division physics courses (e.g. [1, 2]). Course work at the upper-division requires the use of skills that students are expected to have begun developing in their introductory level courses. One such skill is the ability to perform and interpret vector operations (e.g. summing of vector quantities, and scalar and cross products). A lack of proficiency with this skill can interfere with students' learning of physics concepts [3].

In order to help students develop vector competence, it is important to understand the difficulties students have working with the various vector operations. To this end, a number of studies at the introductory level have investigated students' ability to add and subtract vectors (e.g.[3-5]), and students' understanding and execution of vector decomposition and vector products $[6,7]$.

At the upper-division, there is added complexity to performing vector operations when we introduce new coordinate systems. Students are expected to choose an appropriate coordinate system for the context they are working in and are often required to move between coordinate systems. In this paper, we expand the body of knowledge surrounding students' use of vectors by investigating students' approaches to decomposing vectors in Cartesian and polar coordinate systems and identifying student difficulties therein.

\section{DATA}

The data presented in this study come from student $(\mathrm{N}=463)$ responses to one item on the Colorado Classical Mechanics/Math Methods Inventory (CCMI) administered at 10 institutions. The CCMI is a research-based open-ended assessment designed to measure student performance on a set of key skills in upper-division classical mechanics [8]. One item involves the decomposition of a velocity vector into both Cartesian and polar coordinates (shown in Figure 1). It was designed to assess the learning goal: "Students should be able to project a given vector into components in multiple coordinate systems".

\section{METHODS}

The open-ended nature of the question allowed for contextual written information, which provided opportunity for deeper insight into students' approaches to the problem. However, the open-endedness also resulted in a large variance in the completeness and characteristics of the written solutions given by students. In fact, there were numerous instances where students only wrote the velocity expressions without demonstrating any workings of their solution.

In order to capture all student responses, we developed two categorization schemes for both the Cartesian and the polar coordinate systems. One scheme categorizes students' velocity expression. Categories emerged based on the common errors seen in students' final expressions. The categories are exclusive and expressions that contained multiple errors were placed in a category that represented the most central error in that expression. Examples for each coordinate system are shown in Sections IV and V. The other scheme categorizes students' approach to constructing the velocity expression. Categories were determined through additional features of students' responses, such as diagrams and visible mathematical manipulations. In some cases, single features were insufficient to infer an approach. Discussions during category identification and refinement established the required evidence for approach categorizations.

The goal of our analysis was not to document the prevalence of errors made by students in their decomposition of the velocity vector. Instead, we aimed to identify productive approaches that students use to decompose vectors. For this reason it was important for us to consider what errors were more central, i.e. which errors made the expression further from being correct. Also, the exclusivity of the expression categories provided the means to couple final expressions with the approach taken.

The development of all sets of categories began with the individual examination of a small sample $(\mathrm{N} \sim 30)$ of student 


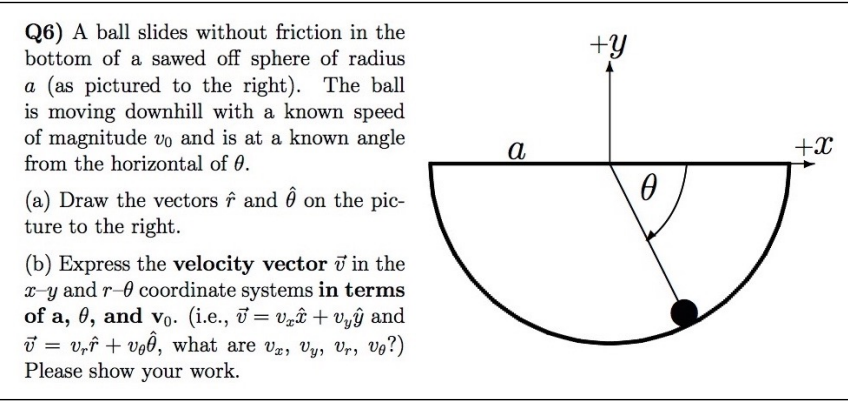

FIG. 1. CCMI item on vector decomposition in multiple coordinate systems.

responses by two researchers. This resulted in a preliminary categorization that was refined through two more iterations using similarly sized samples. Discrepancies in categorization were discussed and resolved after each iteration to improve the reliability of further categorizations. $92 \%$ agreement was obtained for the remainder of student responses.

Only categories that account for at least $10 \%$ of student responses are presented as independent categories. In all categorizations, categories that represent less than $10 \%$ of responses are collapsed into an 'Other' category. Out of our 463 responses, there were 78 students who did not attempt to write an expression for the velocity in either coordinate system. Hence, our categorizations were carried out using a maximum of 385 student responses.

\section{CARTESIAN EXPRESSION}

In this section, we present our categorization of students' Cartesian expressions for the velocity vector and their approaches to determining this expression. We also discuss the intersection between the approach used by students and their resulting Cartesian velocity expression.

Expression categorization: Students' Cartesian expressions were placed into six categories. These categories are shown in Table I along with examples and the distribution of responses in each category. Almost one quarter of students wrote a fully correct Cartesian expression of the velocity vector. A further $21 \%$ had represented the magnitude of both components correctly but had a sign error for one or both components. In $15 \%$ of the final expressions, the trigonometric identities of the components were reversed. In $12 \%$ of the velocity vector expressions, the magnitude of the position vector, $a$, was used instead of the magnitude of the velocity. Within this category, some expressions used the trigonometric identities that correctly corresponded to the components of the velocity vector and others used the trigonometric identities that correctly corresponded to the components of the position vector. The 'Other' category comprises $20 \%$ of student responses. Within this category there are some commonalities among the final expressions like the presence of both the position and velocity vector magnitudes or the absence of either vector. However, individual subcategories
TABLE I. Categories of students' Cartesian expression with examples and the distribution for $\mathrm{N}=385$.

\begin{tabular}{lcc} 
Category & Example & $\mathbf{N}(\%)$ \\
\hline Correct & $-v_{0} \sin \theta \widehat{x}-v_{0} \cos \theta \widehat{y}$ & or \\
& $-a \dot{\theta} \sin \theta \widehat{x}-a \dot{\theta} \cos \theta \widehat{y}$ & \\
\hline Sign error only & $v_{0} \sin \theta \widehat{x}+v_{0} \cos \theta \widehat{y}$ & $82(21)$ \\
\hline Reverses trig identities & $v_{0} \cos \theta \widehat{x}-v_{0} \sin \theta \widehat{y}$ & $58(15)$ \\
\hline Position instead of velocity & $\begin{array}{c}a \sin \theta \widehat{x}+a \cos \theta \widehat{y} \text { or } \\
a \cos \theta \widehat{x}+a \sin \theta \widehat{y}\end{array}$ & $47(12)$ \\
\hline Other & $v_{x}=\sqrt{a^{2}-y^{2}}$, & $75(20)$ \\
\hline None & $v_{y}=\sqrt{a^{2}-x^{2}}$ & \\
\hline
\end{tabular}

such as these accounted for less than $3 \%$ of responses. As described in Section III, each category represents the most central error in students' velocity expression. For example, the expression $a \sin \theta \widehat{x}+a \cos \theta \widehat{y}$ contains a sign error but it is placed in the 'Position instead of velocity' category because we considered using the magnitude of the position vector instead of the velocity vector to be a more serious error than a sign error.

Approach categorization: Our categorization of student approaches to determining the Cartesian expression of the velocity vector is shown in Table II and an example of each approach from student responses is shown in Figure 2. 31 students did not write a Cartesian velocity expression, therefore our categorization of approaches included for 354 responses.

The most frequent approach we identified was the decomposition of the velocity vector (shown in Figure 2(a)). In student responses, this approach was evidenced by the drawing of vertical and horizontal components (labeled or unlabeled) on the $v_{0}$ vector (or the $\widehat{\theta}$ unit vector) and the angle between the $v_{0}$ vector and the vertical component being marked $\theta$.

Another common approach students used was the differentiation of position (shown in Figure 2(b)). Student responses in this category started with a position component expression and then computed the derivative, as evidenced by an expression starting with $\dot{r}$ or an equivalent. In some cases, students showed the decomposition of the given position vector, while in other responses, it seemed that students were recalling the position expressions (e.g. starting with $x=r \cos \theta$, $y=r \sin \theta)$.

Other approaches employed by students (e.g. a manipulation of Pythagoras' theorem) are combined in the Other category as they are each present in only a small number of student responses $(\mathrm{N}<10)$. (Figure $2(\mathrm{c})$ )

For half of the responses, we could not determine the approach taken. $37 \%$ of students wrote an expression, but did not demonstrate in any identifiable way how they reached that answer. A further $13 \%$ had some feature to their response, in addition to their Cartesian expression, but on their own, they did not provide enough evidence to infer an approach. The most common feature to only be accompanied by the Cartesian expression was a drawn vertical line between the ball and the $\mathrm{x}$-axis completing a right-angled triangle with the position vector. (Figure $2(\mathrm{~d})$ ) 
TABLE II. Categories of students' approaches to expressing the velocity vector in the Cartesian coordinate system $(\mathrm{N}=354)$.

\begin{tabular}{lc} 
Category & $\mathbf{N}(\%)$ \\
\hline Velocity vector decomposition & $94(27)$ \\
\hline Position derivative & $48(14)$ \\
\hline Other & $35(10)$ \\
\hline Insufficient evidence & $46(13)$ \\
\hline No evidence & $131(37)$ \\
\hline
\end{tabular}

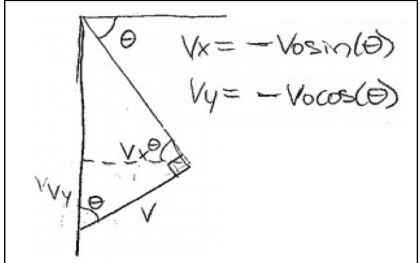

(a) Velocity Decomposition

$\hat{r}=\sqrt{x^{2}+y^{2}}$
$\sqrt{r^{2}-y^{2}=x}$
$\vec{v}=\sqrt{a^{2}-y^{2}} \hat{x}+\sqrt{a^{2}-x^{2}} \hat{y}$
(c)Other

FIG. 2. Examples of student responses in each of the approach categories.

Intersection between Cartesian velocity expression and approach: To investigate the intersection between students' approaches and their Cartesian expression, we removed responses where we had no evidence of approach. Figure 3 shows the distribution of approaches within each Cartesian velocity expression category $(N=223)$. Of the two viable approaches we observed in student responses, decomposing the velocity vector appears to be the most productive. Students who decomposed the velocity vector were more than twice as likely to answer correctly or with only a sign error than if they differentiated the position vector.

When using the differentiation approach, many students did not apply the chain rule. For example, when starting with $\vec{r}=a \cos \theta \widehat{x}+a \sin \theta \widehat{y}$ these students found $\dot{\vec{r}}=-a \sin \theta \widehat{x}+$ $a \cos \theta \widehat{y}$, an expression consistent with the 'Position instead of velocity' category. After finding this expression, some students multiplied each component by $v_{0}$ (a subcategory in the 'Other' category). We cannot tell whether this difficulty with the differentiation is a result of students not knowing when or how to apply the chain rule, or if students did not consider or recognize what variable they were differentiating with respect to.

Decomposing the velocity vector also had some difficulties associated with it, the most prevalent of which was geometric in nature. Students identified the $\theta$ angle incorrectly as the angle between the velocity vector and its horizontal component, resulting in a velocity expression that has trigonometric

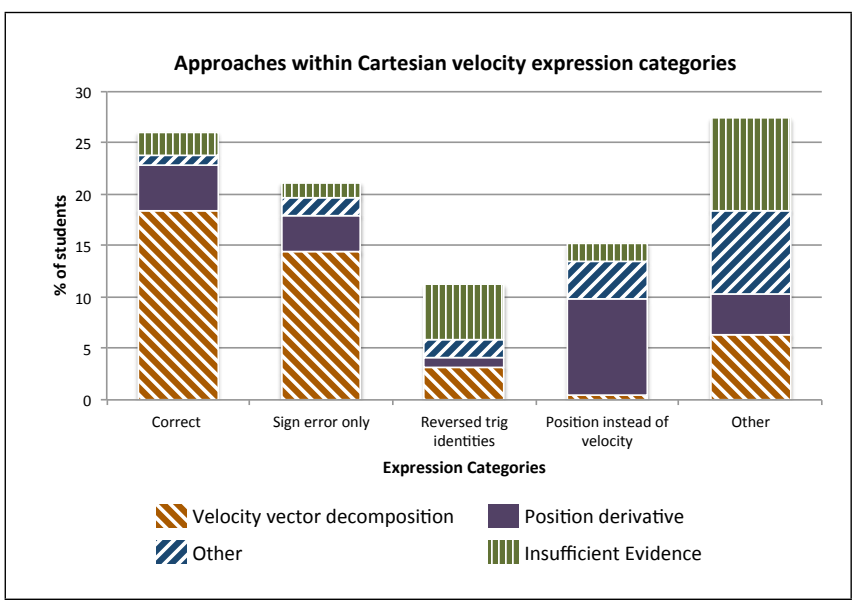

FIG. 3. Approach distribution within each final answer category $(\mathrm{N}=223)$.

identities for each component reversed.

A small number of students showed the decomposition of the position vector and then used that expression for their velocity expression (subcategory in 'Other' approach category), resulting in a 'Position instead of velocity' expression. Others showed the decomposition of the position vector and then replaced the magnitude of the position vector with the magnitude of the velocity vector for their velocity vector expression (another subcategory in the 'Other' approach category), resulting in a 'Reversed trigonometric identities' expression. Though the completing of a triangle on the position vector is insufficient on its own to infer an approach, its prevalence in the 'Reversed trigonometric identities' category suggests that these 'Other' subcategories approaches might actually have been more frequently used by students.

\section{POLAR EXPRESSION}

In this section, we present our categorization of students' polar expressions for the velocity vector and their approaches to determining this expression. We also discuss the intersection between students' polar and Cartesian velocity expressions.

Expression categorization: Students' polar expressions were placed into four categories. These categories are shown in Table III along with examples and the distribution of responses in each category. $40 \%$ of students wrote a correct polar expression of the velocity vector. A further $23 \%$ of students recognized that the radial component of the velocity is zero, but they either did not represent the angular component or determined its magnitude to be something other than $v_{0}$ or $a \dot{\theta}$. There was a large variance in how students expressed the angular component. The most common expressions represented the magnitude as $\dot{\theta}$ or included a trigonometric identity. However, each of these observed expressions accounted for less than $4 \%$ of student responses. $21 \%$ of students wrote an expression with an incorrect magnitude for 
TABLE III. Categories of students' polar expression with examples and the distribution for $\mathrm{N}=385$.

\begin{tabular}{lcc} 
Category & Example & $\mathbf{N}(\%)$ \\
\hline Correct & $0 \widehat{r}+v_{0} \widehat{\theta}$ or $0 \widehat{r}+a \dot{\theta} \widehat{\theta}$ & $155(40)$ \\
\hline$v_{r}=0$ & $0 \widehat{r}+\dot{\theta} \widehat{\theta}$ or $0 \widehat{r}+v_{0} \sin \theta \widehat{\theta}$ & $88(23)$ \\
\hline$v_{r} \neq 0$ & $a \widehat{r}+\dot{\theta} \widehat{\theta}$ or $a \sin \theta \widehat{r}+a \cos \theta \widehat{\theta}$ & $81(21)$ \\
\hline None & & $61(16)$ \\
\hline
\end{tabular}

both the radial and angular components. Responses in this category were even more varied. The radius of the sphere, $a$, was the most common magnitude used for the radial component. Similar to the ' $v_{r}=0$ ' category, trigonometric identities were sometimes used in both components.

Approach categorization: A categorization of student approaches to determining the polar velocity vector expression, similar to that for the Cartesian expression, was not possible to develop. The vast majority of students did not show how they reached their polar expression.

However, approximately $30 \%$ of students who answered correctly provided explanations for why the radial component is zero (in some cases, this was combined with an explanation for the magnitude of the angular component). These explanations suggest that students were approaching the writing of the polar expression in two different ways. One approach seems to be conceptual in nature, where students considered the direction of motion of the ball and compared that to the direction of the radial and angular directions, e.g. "the ball is not moving in $\widehat{r}$ direction, all the velocity must be in the $\widehat{\theta}$ direction" or "velocity is tangential to the sphere". The second approach seems to be more mathematical, where students consider the changing position of the ball, e.g. " $v_{r}=\dot{r}=$ zero because $r$ is not changing therefore velocity must all be in $\widehat{\theta}$ ". We observed similar explanations for why the magnitude of the radial component is zero in the $v_{r}=0$ category, though a smaller percentage of students provided explanations within this category. We do not have enough evidence to suggest which of these approaches might be more productive.

Polar and Cartesian velocity expressions intersection: Due to the absence of an approach categorization in polar, we can only discuss comparisons between the Cartesian and polar velocity expression categories. A similar number of students $(\sim 40 \%)$ successfully expressed the velocity in each coordinate system (neglecting a sign error). However, only $19 \%$ of students were successful in both coordinate systems. This suggests that competency in one system does not necessarily translate to the other.

Students' expression for the polar expression were much more varied than for the Cartesian expression. Also, twice as many students did not write a polar expression. A possible explanation for these differences might be that many students in intermediate mechanics have more solid resources surrounding details of the Cartesian system, while often having to derive details of the polar system [9].

\section{CONCLUSION}

Our results show that student difficulties with vector decomposition persist from introductory level to the upperdivision, with less than $20 \%$ of students successfully expressing a vector in both the Cartesian and polar coordinate systems. Students demonstrated more systematic approaches to determining components in the Cartesian coordinate system. Our analysis also opened many questions that we were unable to investigate given the nature of our written response data. For example, why do students choose deriving position as an approach to expressing Cartesian velocity components? And how do students approaches to vector decomposition vary for different coordinate systems? In future work, we plan to investigate these specific approach questions using an interview observation methodology.

\section{ACKNOWLEDGMENTS}

We gratefully acknowledge Lyman Briggs College and the MSU Honors College for supporting this work. We would also like to thank the members of PERL@MSU for their useful comments and suggestions at various stages of this work.
[1] B. S. Ambrose, Investigating student understanding in intermediate mechanics, AJP 72, 453 (2004).

[2] C. Singh et al., Improving students' understanding of quantum mechanics, Physics Today 59, 43 (2006).

[3] N.-L. Nguyen and D. E. Meltzer, Initial understanding of vector concepts among students in introductory physics courses, AJP 71, 630 (2003).

[4] S. Flores et al., Student use of vectors in introductory mechanics, AJP 72, 460 (2004).

[5] A. F. Heckler and T. M. Scaife, Adding and subtracting vectors: The problem with the arrow representation, PRST-PER 11 (2015).
[6] J. Van Deventer and M. C. Wittmann, Comparing student use of mathematical and physical vector representations, PERC Proc. (2007).

[7] G. Zavala and P. Barniol, Students understanding of the concepts of vector components and vector products, PERC Proc. (2010).

[8] M. D. Caballero and S. J. Pollock, Assessing student learning in middle-division classical mechanics/math methods, PERC Proc. (2014).

[9] E. C. Sayre and M. C. Wittmann, Plasticity of intermediate mechanics students coordinate system choice, PRST-PER 4 (2008). 